\title{
Effect of Stimulation of Sublobule IX-b of the Cerebellar Vermis on Cardiac Function
}

\author{
I. ROCHA, V. GONÇALVES, M. J. BETTENCOURT, L. SILVA-CARVALHO \\ Institute of Physiology, Faculty of Medicine of Lisbon and Unit of Autonomic Nervous System, \\ Institute of Molecular Medicine, Lisbon, Portugal
}

Received April 4, 2007

Accepted July 9, 2007

On-line October 11, 2007

\section{Summary}

Activation of sublobule IX-b of the cerebellar vermis evokes hypotension, bradycardia and decrease of the phrenic nerve activity in the anesthetized animal. Cardiac performance during the isovolumic phases of systole and relaxation can be evaluated by $\mathrm{dP} / \mathrm{dt}_{\max }, \mathrm{Vpm}, \mathrm{dP} / \mathrm{dt} / \mathrm{DP}_{40}$ and $\mathrm{T}$, respectively. In the present study, we evaluated the changes on cardiac function evoked by the stimulation of sublobule IX-b. New Zealand white rabbits were anesthetized, paralyzed and artificially ventilated. A posterior craniotomy was made to reveal and stimulate the cerebellar uvula ( $4 \mathrm{~s}$ train; $50 \mathrm{~Hz} ; 1 \mathrm{~ms} ; 20 \mu \mathrm{A}$ ). The femoral artery and veins were cannulated and a Swan-Ganz catheter was advanced in the upper abdominal aorta to control afterload when inflating the balloon. The left ventricle was catheterized with a Millar catheter. Blood pressure, heart rate, left ventricular pressure were monitored. Results showed a significant decrease on sublobule IX-b stimulation of all the indices of systolic function and an increase of $T$ indicating a decrease in the speed of the relaxation. These data provide the first evidence of the influence of sublobule IX-b on cardiac function. They may contribute to the understanding of the origin the cardiovascular changes that were observed in two patients with vermian and paravermian hemorrhage.

\section{Key words}

Cerebellum • Autonomic nervous system • Vermis • Cardiac function

\section{Corresponding author}

Isabel Rocha, Instituto de Fisiologia, Faculdade de Medicina de Lisboa, Av. Prof. Egas Moniz, 1649-028 Lisbon, Portugal. Fax: +351 217999436. E-mail: isabelrocha@fm.ul.pt

\section{Introduction}

The assessment of ventricular function is an important task in the evaluation of patients with known or suspected heart disease. There are several parameters that can be used to evaluate ventricular function but most of them are relatively dependent on afterload and preload. The maximum rate of rise of ventricular pressure (dP/dtmax), largely independent of afterload changes provided these occur before aortic valve opening, is influenced both by large changes of preload and by acute changes of contractility (Gleason and Braunwald 1962). An index that is less affected by preload and is not affected by afterload is $\mathrm{dP} / \mathrm{dt} / \mathrm{DP}_{40}$ which is the ratio between $\mathrm{dP} / \mathrm{dtmax}$ and the developed ventricular pressure computed at a DP of $40 \mathrm{~mm} \mathrm{Hg}$ (Braunwald 1988), where developed pressure (DP) is defined as the left ventricular pressure minus the end-diastolic pressure. To access directional changes of contractility, another index relatively independent of afterload and preload, the peak of $\mathrm{dP} / \mathrm{dt} / \mathrm{TP}$ (TP being the total pressure development), which is also termed as Vpm, can be applied (Braunwald 1988). Previous work by several authors had shown that the time course of the fall of left ventricular pressure after $\mathrm{dP} / \mathrm{dtmin}$ has an exponential profile that defines an index $-\tau-$ which allows the characterization of the isovolumic relaxation phase (Leite-Moreira 1997, Weiss et al 1976).

Stimulation of sublobule IX-b of the posterior vermis of the cerebellum provokes in the anesthetized animal a cardiovascular response characterized by hypotension and bradycardia, and an accompanying decrease of phrenic nerve activity (Bradley et al 1987a). Previous studies (Bradley et al 1987a, b, Gonçalves et al 
2002, Rocha et al. unpublished observations) have shown that this depressor response is due to a decrease in sympathetic activity rather than due to an increase of the parasympathetic outflow. As yet no data are available relating the activation of sublobule IX-b to cardiac function, and in particularly to inotropic state.

In a previous study, we reported a clinical case of a cerebellar hemorrhage as a cause of a neurogenic pulmonar edema (Gonçalves et al 2005). We suggested that the observed cerebellar vermian and paravermian hemorrhage compressing the cerebellar uvula was the cause of the initial sympathetic storm that elicited the observed tachycardia and a marked rise in blood pressure that preceded the acute pulmonary edema. In a recently published clinical case Deininger et al. (2006) report changes on cardiac function of a patient with no history of cardiac disease who suffered progressive tachycardia, fibrillation and electromechanical decoupling with the rupture of an angioma of the cerebellar vermis. Thus, the purpose of the present work was to study the changes elicited on cardiac function by the activation of sublobule IX-b of the cerebellum of the anesthetized rabbit. A preliminary report of this work has been published in abstract form (Gonçalves et al. 2006).

\section{Methods}

\section{Anesthesia, surgical protocol}

Nine New-Zealand white rabbits $(2.7-3.2 \mathrm{~kg})$ were anesthetized with sodium pentobarbitone $(40 \mathrm{mg} / \mathrm{kg}$ ip) supplemented as required. The depth of the anesthesia was assessed by pinching a paw before neuromuscular blockade with pancuronium bromide, $(4 \mathrm{mg} / \mathrm{kg} / \mathrm{h})$ and by observing changes on arterial blood pressure (BP) and heart rate (HR) after blockade. A tracheotomy was made low in the neck to allow the insertion of a tracheal cannula for artificial ventilation with $\mathrm{O}_{2}$-enriched air (rate of ventilation $50-55 \mathrm{cpm}$ for an end-tidal $\mathrm{CO}_{2}$ of $4.5-5 \%$ ). The left femoral vein was cannulated for injecting drugs or saline. Through the femoral artery was introduced a Swan-Ganz catheter (4F), the tip of which was placed in the abdominal aorta, for measuring BP and also to evoke afterload increases by inflating its balloon. The urinary bladder was cannulated and drained to avoid inhibition of cardiorespiratory reflexes (Daly 1997). The right common carotid artery was catheterized at cervical level and through it a Millar micro-tip catheter (Millar, USA) was introduced, under RX control (BV300, Philips), in the left ventricle. The confirmation of the location of its tip was made by the profile of the blood pressure curve observed. The rectal temperature was maintained at 37.5$39^{\circ} \mathrm{C}$ by a servo controlled heating blanket (Harvard Apparatus Ltd). The electrocardiogram (ECG) was recorded (Neurolog, Digitimer) with the use of needle electrodes inserted into the limbs and heart rate derived with the use of an instantaneous ratemeter (Neurolog, Digitimer). The animal's head was placed in a stereotaxic frame (Kopf Instruments) and a craniotomy was performed to expose the uvula and to allow the insertion of a double-barreled glass microelectrode for electrical stimulation of sublobule IX-b using the barrel filled with Woods metal, (4 s train; $50 \mathrm{~Hz} ; 1 \mathrm{~ms} ; 20 \mu \mathrm{A}-$ submaximal stimulations) and, using the second barrel and for labeling the stimulated sites with pontamine sky blue dye $(2 \%)$ in sodium acetate ( $1 \mathrm{M})$. Arterial blood pressure (BP), left ventricular pressure (LVP) and ECG were monitored (Neurolog, Digitimer). At the end of the experiment, animals were killed with an overdose of anesthetic. All the procedures using animals were performed according to national and E.U. laws on animal experimentation and the principles of laboratory animal care.

\section{Experimental protocol}

Sublobule IX-b was identified using electrical stimulation to elicit its characteristic cardiovascular depressor responses - hypotension and bradycardia (supramaximal stimulation - $4 \mathrm{~s}$ train; $50 \mathrm{~Hz} ; 1 \mathrm{~ms}$; $>50 \mu \mathrm{A})$. After the correct placement of the electrode, one submaximal stimulation $(\leq 20 \mu \mathrm{A})$ was performed during which ventilation was suspended. After stimulation and recovery to baseline conditions a prolonged period of electrical stimulation (15s) was performed during which, and as soon as blood pressure begun to fall, the Swan-Ganz balloon that had been placed in the abdominal aorta was inflated in order to simulate an increase in afterload. During this period of $15 \mathrm{~s}$ the ventilation was suspended. The volume of the inflated balloon was minimized to maintain blood pressure in the same range of values that was before the beginning of electrical stimulation. This balloon was kept inflated during $5 \mathrm{~s}$ and, after $5 \mathrm{~s}$ of its disinflation, electrical stimulation was switched-off.

\section{Histology}

After the labeling with deposition of pontamine sky blue of the stimulation sites at the end of the experiment, the cerebellum was removed and fixed in a 
$4 \%$ paraformaldehyde saline with $30 \%$ sucrose solution for 48 hours. The tissue was sectioned serially $(80 \mu \mathrm{m})$ and stained with neutral red. Stimulating sites within the cerebellar uvula were identified according to the rabbit atlas by Meesen and Olszewski (1949).

\section{Signal acquisition and data analysis}

All recorded variables were digitized (Instrutech VR100B, Digitimer Ltd) and recorded on video-tape. Off-line analysis was done using a PowerLab system computer and analysis software (PowerLab).

For the variables recorded (BP, LVP and heart rate), baseline values were taken immediately before the beginning of the stimulation (control). These values were compared with those obtained at the peak of the response evoked by the stimulation (Stim). From LVP values $\mathrm{dP} / \mathrm{dtmax}, \mathrm{Vpm}, \mathrm{dP} / \mathrm{dt} / \mathrm{DP}_{40}$ and $\tau$ were calculated. The index $\tau$ was calculated by the derivative method (Weisfeldt et al. 1978, Weiss et al. 1976). Briefly, this method is based on the mathematical principles that a derivative of an exponential function is also exponential and when an exponential function is expressed by other exponential function a linear relation is obtained. That is, the fall in ventricular pressure which is described by an exponential function is transformed in an equation of a line and $\tau$ will correspond to the symmetrical of the inverse of the slope of the calculated line equation.

For statistical analysis the $t$-Student test for paired observations was used and values of $t$ were considered significant when $\mathrm{p}<0.05$. All data are expressed as mean $\pm \mathrm{SD}$.

\section{Results}

Before any electrical stimulation, the baseline values of mean blood pressure (BPm), heart rate (HR) and maximum left ventricular pressure (LVPmax) were $101 \pm 9.3 \mathrm{~mm} \mathrm{Hg}, 229 \pm 12 \mathrm{bpm}$ and $133 \pm 11.5 \mathrm{~mm} \mathrm{Hg}$, respectively. Electrical stimulation of the uvula $(4 \mathrm{~s}$ train; $50 \mathrm{~Hz} ; 1 \mathrm{~ms} ; 20 \mu \mathrm{A})$ elicited the characteristic cardiovascular response - bradycardia and hypotension showing a significant decrease of BPm, HR and LVPmax to $79 \pm 7.0 \mathrm{~mm} \mathrm{Hg}, 204 \pm 8.0 \mathrm{bpm}$ and $108 \pm 13.8 \mathrm{~mm} \mathrm{Hg}$ respectively $(n=9, p<0.05)$ (Fig. 1$)$.

The computation of $\mathrm{dP} / \mathrm{dtmax}$ showed a significant decrease during systole after stimulation of sublobule IX-b as dP/dtmax decreased from $2038 \pm 81.7$ to $1675 \pm 118.8 \mathrm{~mm} \mathrm{Hg} \mathrm{s}^{-1}$ (Fig. 1, Table 1) while $\tau$ increased significantly from $10.9 \pm 1.50$ to $13.1 \pm 1.70 \mathrm{~ms}$ (Fig. 2,

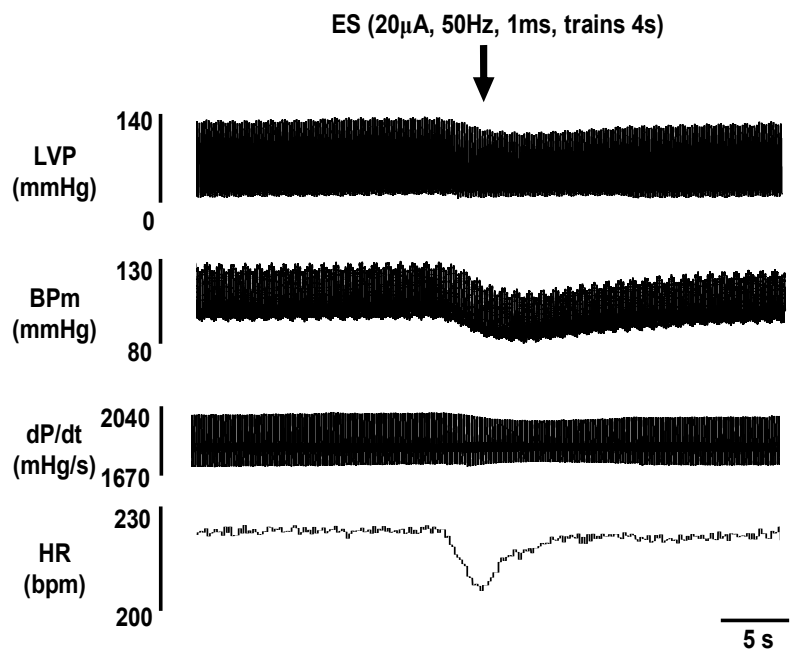

Fig. 1. Effect of electrical stimulation of sublobule IX-b of the cerebellar vermis showing the characteristic depressor responsehypotension and bradycardia accompanied by a decrease in maximum $\mathrm{dP} / \mathrm{dt}$ which indicates a decrease in the rate of the myocardial fibers during systole (BPm - mean blood pressure, LVP - left ventricular pressure, HR - heart rate)

Table 1) which indicates that sublobule IX-b stimulation evokes a decrease of the rate of isovolumic relaxation $(\mathrm{n}=9, \mathrm{p}<0.05)$. The values of $\mathrm{Vpm}$ and $\mathrm{dP} / \mathrm{dt} / \mathrm{DP}_{40}$ also decreased from $37 \pm 3.3$ to $30 \pm 4.9 \mathrm{~s}^{-1}$ and from $34 \pm 1.0$ to $29 \pm 0.6 \mathrm{~s}^{-1}$, respectively $(\mathrm{n}=9, \mathrm{p}<0.05$, Table 1$)$. In relation to end-diastolic pressure no significant modifications were observed as values changed from $7.4 \pm 1.51$ (basal period) to $8.1 \pm 2.28 \mathrm{mmHg}$ (on stimulation) $(n=9, p=0.06)$. Furthermore, during the inflation of the indwelling Swan-Ganz balloon placed in the abdominal aorta which increased afterload and brought LVP on stimulation to values similar to those observed during basal condition, the calculation of $\mathrm{dP} / \mathrm{dt}$ showed a significant decrease to $1840 \pm 70.5 \mathrm{~mm} \mathrm{Hg} \mathrm{s}^{-1}$ $(\mathrm{n}=9, \mathrm{p}<0.05)$. Under these conditions, heart rate was $200 \pm 91 \mathrm{bpm}$ and end-diastolic pressure was $8.3 \pm 2.5$ $\mathrm{mm} \mathrm{Hg}$.

\section{Discussion}

The primary result of this study is to show that the activation of the sublobule IX-b of the cerebellar uvula evokes changes in cardiac function. Furthermore, these results provide indications of the putative origin of the cardiovascular signs observed in patients that had a vermian hemorrhage with compression of the cerebellar uvula.

End-diastolic fiber length, myocardial contractility (inotropism) and relaxation (lusitropism) are 
Table 1. The values of the computed indexes of ventricular function. The significant decrease of $d P / d t m a x, V p m$ and $d P / d t / T P$ indicate a decrease in the speed of contraction of the myocardial fibers during the isovolumic phase of systole while $\mathrm{T}$, by its significant increase, shows a decrease in the speed of relaxation during the isovolumic phase of diastole $(n=9 ; p<0.05$; data expressed as mean \pm SD). The previous changes occurred without significant changes of end-diastolic pressure ( $n=9, p=0.06$, (NS- non-significant)).

\begin{tabular}{|c|c|c|}
\hline Parameters & Control values & Values on stimulation \\
\hline dP/dtmax $\left(m m H g s^{-1}\right)$ & $2038 \pm 81.7$ & $1675 \pm 118.8$ \\
\hline $\operatorname{Vpm}\left(s^{-1}\right)$ & $37 \pm 3.3$ & $30 \pm 4.9$ \\
\hline$d P / d t / D P\left(s^{-1}\right)$ & $34 \pm 1.0$ & $29 \pm 0.6$ \\
\hline$\tau(m s)$ & $10.9 \pm 1.50$ & $13.1 \pm 1.70$ \\
\hline End-diastolic pressure ( $\mathrm{mm} \mathrm{Hg}$ ) & $7.4 \pm 1.51$ & $8.1 \pm 2.28(\mathrm{NS})$ \\
\hline
\end{tabular}

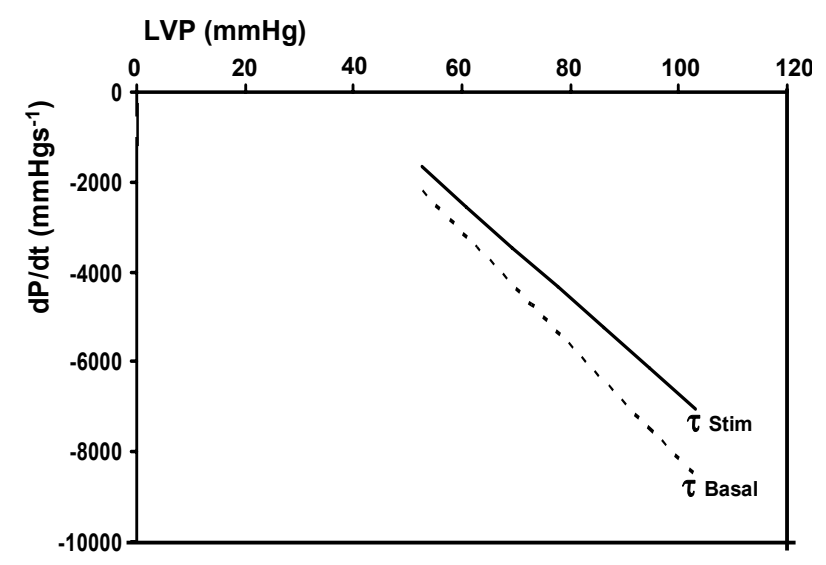

Fig. 2. The rate of isovolumic relaxation decreases during the stimulation of sublobule IX-b as is shown by the rise of the slope of the lines representing basal (TBasal) and stimulation conditions (TStim) taken from one representative animal.

the determinants of cardiac ejection and filling and their assessment could be made by parameters that describe contractile function and relaxation.

Most of the parameters, that describe the contractile function, are based on the analysis of pressure measurements obtained during isovolumic contraction and their major advantage is that the data are collected before the opening of the aortic valve (Katz 2001). The majority of these parameters is dependent on the load but $\mathrm{dP} / \mathrm{dtmax}$ that represents the peak rate of the rise of left ventricular pressure is mainly influenced by contractility (Braunwald 1988, Little 1987), although regional abnormalities in left ventricular function and the size and thickness of the left ventricle can also affect this parameter (Katz 2001). Changes in dP/dtmax are known to be sensitive to acute changes in contractility together with end-diastolic volume and filling pressure, so that $\mathrm{dP} / \mathrm{dtmax}$ can be used for evaluation of the directional changes in contractility when performing an intervention (Leite-Moreira 1997). Other indices of cardiac function,

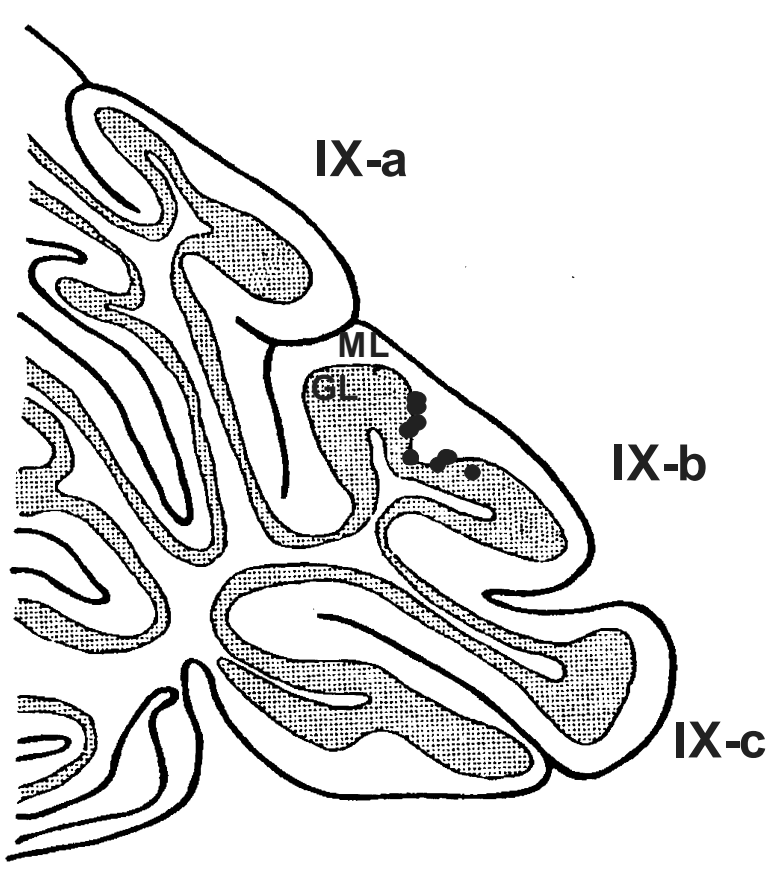

$600 \mu \mathrm{m}$

Fig. 3. The stimulation sites $(n=9)$ restricted to the sublobule IX-b of the vermis ( $\mathrm{ML}$ - molecular layer; $\mathrm{GL}$ - granular layer).

that are based on events occurring in the isovolumetric phase of the cardiac cycle, are either $\mathrm{dP} / \mathrm{dt} / \mathrm{DP}_{40}$ where DP is the developed left ventricular pressure (i.e. left ventricular pressure minus end-diastolic pressure) computed at a DP of $40 \mathrm{~mm} \mathrm{Hg}$, or peak $\mathrm{dP} / \mathrm{dt} / \mathrm{TP}$, also termed as Vpm, where TP refers to the total pressure development. Other index is Vmax, which corresponds to the maximum velocity of shortening of the unloaded contractile elements, but controversy still exist in relation to its calculation both in isolated myocardial fibers and in the intact heart. Despite being independent from preload 
and afterload, Vmax appears to have little advantage over $\mathrm{dP} / \mathrm{dtmax}$ or $\mathrm{dP} / \mathrm{dt} / \mathrm{DP}_{40}$. Conversely, $\mathrm{dP} / \mathrm{dt} / \mathrm{DP}_{40}$ is relatively simple to obtain and has advantage over $\mathrm{dP} / \mathrm{dtmax}$ because is relatively independent of the time and level of arterial pressure in the instant of aortic valve opening; but, despite being insensitive to changes in afterload it increases slightly with larges changes in preload. Vpm is an index that is relatively independent of changes in both afterload and preload (Leite-Moreira 1997), but Katz (2001) found it to be relatively insensitive to changes of afterload but was influenced by preload, decreasing with the increase of left ventricular end-diastolic pressure.

To evaluate relaxation, the maximum rate of fall of left ventricular pressure during isovolumic relaxation $-\mathrm{dP} / \mathrm{dtmax}$ is reasonable, but this index is highly dependent on aortic pressure. Since the decline in left ventricular pressure is often assumed to be exponential with time it allows to express $-\mathrm{dP} / \mathrm{dtmax}$ as an exponential function thus making possible to calculate a time constant $(\tau)$ based on the time required for ventricular pressure to decline to half or $1 / \mathrm{e}$ of its peak pressure, beginning at the time of aortic valve closure (Little 1987).

Accordingly, the three indices of contractile function (dP/dtmax, $\mathrm{dP} / \mathrm{dt} / \mathrm{DP}_{40}$ and $\left.\mathrm{Vpm}\right)$ and the index $\tau$ for analysis of lusitropism were applied in our study. Our results show a significant decrease in cardiac performance as the three indices of systolic function (dP/dtmax, $\mathrm{dP} / \mathrm{dt} / \mathrm{DP}_{40}$ and $\mathrm{Vpm}$ ) decreased on stimulation and the $\tau$ index increased significantly during the activation of sublobule IX-b without significant changes on end-diastolic pressure. Great care was used to minimize the effect of afterload during balloon inflation in the abdominal aorta as the purpose of the inflation during stimulation was to compensate the decrease of pressure evoked by sublobule IX-b stimulation. Under these conditions $\mathrm{dP} / \mathrm{dtmax}$ values decreased significantly during sublobule IX-b stimulation confirming the decrease on the rate of contraction of myocardial fibers during the isovolumic period of systole.

Several animal studies have shown that the sublobule IX-b of the cerebellar vermis is involved in cardiovascular control (Bradley et a, 1987a, b, Gonçalves et al 2002, Paton and Spyer 1992). Animal experiments have provide the evidence for the co-existence of two functionally distinct pathways from the cardiovascular region of sublobule IX-b to the lateral parabracheal nucleus $(\mathrm{PBN})$ of the pons, which is one of the relay stations of the central autonomic network (Paton and Spyer 1990). In particular, sublobule IX-b activation evokes a depressor response, hypotension accompanied by bradycardia, a decrease of respiratory rate and a transient inhibition of the renal sympathetic activity (Bradley et al 1987a, b) in the decerebrate anesthetized animal and this depressor response is mediated by inhibitory Purkinje cells that project from the sublobule IX-b to the rostral lateral PBN (Paton and Spyer 1990). In the decerebrate non-anesthetized animal, a tachycardia together with a pressor response are observed. These cardiovascular responses appear to be mediated by a different neuronal circuit that includes the caudal part of the parabracheal nucleus and its neuronal connections (thought the nucleus tractus solitarius of the medulla (NTS)) with the rostroventrolateral medulla (RVLM) which is the origin of sympathetic outflow to the cardiovascular system (Paton et al. 1990, Paton and Spyer 1992, Silva-Carvalho et al. 1991). We should stress that the pressor/depressor response was reversible in its nature, from the decerebrate to the anesthetized decerebrate animal, when anesthetic was given to the animal (Paton and Spyer 1990). This made the authors to suggest that the neuronal circuit through the NTS was more sensitive to anesthetic action. In conclusion, at the present, the cardiovascular responses from the sublobule IX-b of the cerebellar vermis are presumed to be mediated by two neuronal pathways - one sympathoinhibitory and, the other sympatho-excitatory - that relay at the rostral and caudal PBN, respectively, which allow sublobule IX-b to access the cardiovascular control network and thereby permitting the modulation of peripheral inputs of cardiovascular neurons within both the NTS and RVLM (Paton 1997).

In a previous study (Gonçalves et al. 2005), we reported a case of neurogenic pulmonary edema in a 27-year-old woman caused by a cerebellar hemorrhage due to a vermian and paravermian arteriovenous malformation rupture and we emphasized the involvement of sublobule IX-b, due to its compression by the accumulation of blood, in the increase of sympathetic activity (hypertension and tachycardia) and the observed neurogenic pulmonary edema. Recently (Deininger et al 2006) have reported changes in cardiac function, with modifications of ventricular kinetics similar to those observed on tako-tsubo cardiomyopathy, in a 23-year-old healthy male subject who suffered a four ventricular hemorrhage due to an angioma of the cerebellar vermis.

These novel findings on cardiac function elicited 
by sublobule IX-b of the cerebellum together with data from previous work (Gonçalves et al. 2006, Rocha et al. unpublished observations) (where we showed that during sublobule IX-b activation the observed cardiovascular changes are due to a decrease of sympathetic activity rather that an increase in the parasympathetic outflow) could, at least partially, be used to speculate on the origin of the cardiac changes observed in the two patients, under the conditions comparable with the decerebrate nonanesthetized animal, where clinical reports showed a vermian hemorrhage and-resulting in changes in cardiac function that were, in nature, reversible after the decompression of the lower part of the brainstem.

\section{Conflict of Interest}

There is no conflict of interest.

\section{References}

BRADLEY DJ, GHELARDUCCI B, PATON JFR, SPYER KM: The cardiovascular responses elicited from the posterior cerebellar cortex in the anaesthetized and decerebrate rabbit. J Physiol Lond 383: 537-550, 1987a.

BRADLEY DJ, PASCOE JP, PATON JFR, SPYER KM: Cardiovascular and respiratory responses evoked from the posterior cerebellar cortex and fastigial nucleus in the cat. $J$ Physiol Lond 393: 107-121, $1987 \mathrm{~b}$.

BRAUNWALD E: Assessment of cardiac function. In: Heart Disease - a Textbook of Cardiovascular Medicine. E BRAUNWALD (ed), W B Saunders, New York 1988, pp 449-470.

DALY M DE B: Reflex effects on the cardiovascular system: role of changes on respiration. In: Peripheral Arterial Chemoreceptors and Respiratory Cardiovascular Integration. Clarendon Press, Oxford, 1997, pp 161-181.

DEININGER MH, RADICKE D, BUTTLER J, SCHEUFLER KM, FREIMAN T, ZENTNER JF: Tako-tsubo cardiomyopathy: reversible heart failure with favorable outcome in patients with intracerebral hemorrhage. Case report. J Neurosurg 105: 465-467, 2006.

GLEASON WL, BRAUNWALD E: Studies on the first derivative of the ventricular pressure pulse in man. $J$ Clin Invest 41: 80-91, 1962.

GONÇALVES V, BETTENCOURT MJ, SILVA-CARVALHO L, ROCHA I: Influence of the stimulation of sublobule IX-b of cerebellar cortex on ventricular function in the anaesthetized rabbit. Clin Auton Res 16: 182, 2006.

GONÇALVES V, ROCHA I, SILVA-CARVALHO L: Attenuation of carotid body evoked-responses during the stimulation of the posterior vermis in the anaesthetized rabbit. Auton Neurosci 101: 78-84, 2002.

GONÇALVES V, SILVA CARVALHO L, ROCHA I: Cerebellar haemorrhage as a cause of neurogenic pulmonary edema - case report. Cerebellum 4: 1-4, 2005.

KATZ AM: Physiology of the Heart. Lippincott Williams \& Wilkins, UK, 2001.

LEITE-MOREIRA A: Relaxamento miocárdico normal e patológico. Estudo experimental no coração in-situ. PhD Thesis, Faculty of Medicine of Oporto, Portugal, 1997.

LITTLE WC: The left ventricular $\mathrm{dP} / \mathrm{dt}_{\max }-$ end-diastolic volume relaxation in closer-chest dogs. Circ Res 56: 808-815, 1987.

MEESEN H, OLSZWESKI J: A Cytoarchitectonic Atlas of the Rhombencephalon of the Rabbit. Karger, New York, Basel, 1949.

PATON JFR, SILVA-CARVALHO L, THOMPSON CS, SPYER KM: Nucleus tractus solitarius as mediator of evoked parabrachial cardiovascular responses in the decerebrate rabbit. J Physiol Lond 428: 693-705, 1990.

PATON JFR, SPYER KM: Brain stem regions mediating the cardiovascular responses elicited from the posterior cerebellar cortex in the rabbit. J Physiol Lond 427: 533-552, 1990.

PATON JFR, SPYER KM: Cerebellar cortical regulation of circulation. News Physiol Sci 7: 124-129, 1992.

PATON JFR: Cerebellar control of cardiovascular and respiratory activity. In: Central Nervous Control of Autonomic Function. G BURNSTOCK (ed), Harwood Academic Publishers, UK, 1997, pp 225-258

RAFF GL, GLANTZ SA: Volume loadings slows left ventricular isovolumic relaxation rate. Circ Res 48: 813-824, 1981.

ROCHA I, GONÇALVES V, LARANJO S, DUCLA-SOARES E, ANDRADE A, BOTO JP, SILVA-CARVALHO L: Effect of stimulation of sublobule IX-b of the cerebellar vermis on autonomic outflow to the cardiovascular system: a wavelet analysis. (submitted) 
SILVA-CARVALHO L, PATON JF, GOLDSMITH GE, SPYER KM: The effects of electrical stimulation of lobule $\mathrm{IXb}$ of the posterior cerebellar vermis on neurones within the rostral ventrolateral medulla in the anaesthetised cat. J Auton Nerv Syst 36: 97-106, 1991.

WEISFELDT ML, FREDERIKSEN JW, YIN FC, WEISS JL: Evidence of incomplete left ventricular relaxation in the dog: prediction from the time constant for isovolumic pressure fall. J Clin Invest 62: 1296-1302, 1978.

WEISS JL, FREDERIKSEN JW, WEISFELDT ML: Hemodynamic determinants of the time-course of fall in canine left ventricular pressure. J Clin Invest 58: 751-760, 1976. 